\title{
Experimental free ligand conformations: a missing link in structure-based drug discovery
}

\author{
Elisabetta Chiarparin*,1, Martin J Packer ${ }^{2}$ \& David M Wilson ${ }^{1}$ \\ ${ }^{1}$ Medicinal Chemistry, Oncology, IMED Biotech Unit, AstraZeneca, Cambridge, UK \\ ${ }^{2}$ Medicinal Chemistry, Oncology, IMED Biotech Unit, AstraZeneca, Alderley Park, UK \\ *Author for correspondence: Tel.: +44 781852 4246; Elisbetta.Chiarparin@astrazeneca.com \\ 'G In our experience, it is critical that free ligand conformational information is available to \\ chemists within a timeframe consistent with 'design, make, test, analyze' cycle times of typical \\ drug discovery projects"
}

First draft submitted: 23 September 2018; Accepted for publication: 13 November 2018; Published online: 16 January 2019

Keywords: free ligand conformations $\bullet$ medicinal chemistry $\bullet$ NMR $\bullet$ protein structure $\bullet$ structure-based drug discovery $\bullet$ x-ray

Structure-based drug discovery (SBDD) is a cornerstone of modern medicinal chemistry and has inspired countless small molecule lead optimization efforts and contributed to the discovery of many launched drugs. The number and range of protein structures has increased exponentially in the last decade and now encompass highly dynamic targets previously impervious to crystallography, such as membrane-bound GPCRs and complexes involving proteinprotein interactions (PPI). X-ray structures of protein ligand complexes are generally available with a turnaround time that is compatible with drug discovery cycle times and are typically of sufficient resolution at the atomic-level to inspire effective drug design. However, static protein-ligand interactions observed in $\mathrm{x}$-ray structures are often not sufficient to fully explain structure-activity relationships (SAR) and this can lead to unnecessary synthesis cycles, which ultimately adversely impact project cycle times.

Structure-based drug design is underpinned by the assumption that ligands bind to a protein target via a lockand-key' or 'induced fit' mechanism [1], in which a flexible ligand binds to a rigid protein pocket. More recently, the concept of ligand conformational selection [1] has also emerged, suggesting that the ligand may encounter multiple forms of the protein in solution. Of interest to us in this commentary is the combination of a single conformational state to describe a protein, usually derived from $\mathrm{x}$-ray crystallographic data, with a ligand designed to adopt the required bound state (the bioactive conformation) in solution in the absence of protein. In this context, the ligand is understood to have multiple possible shapes when free in solution, dictated mainly by the number of rotatable bonds and binding is driven by shape complementary [1] between one of its solution state conformers and the binding site within the target protein. The solution phase ensemble from which the bioactive conformation must be selected imposes both entropic and enthalpic penalties on binding. A rigid ligand, which is preorganised into the bioactive conformation, should therefore typically have higher affinity for the target compared with a molecule that has a similar arrangement of functionality but is more flexible and can adopt multiple conformational states in solution. This concept has inspired ligand rigidification strategies that have been used by medicinal chemists to drive target affinity, such as functional group changes, introduction of intramolecular interactions or by macrocyclization [2]. This concept is also covered in a recent publication from Lawson et al. [3], who specifically highlight the impact of ligand rigidity as a strategy in the design of efficient protein-protein interactions inhibitors.

A range of recent structure-based drug discovery case studies demonstrate the power of nuclear magnetic resonance (NMR) conformational analysis to elucidate SAR trends with respect to conformational preferences of the free ligand [4-14]. In the majority of cases, knowledge of the free ligand conformational preference clearly aided structure-based design. Protein x-ray crystallography can provide detailed knowledge on protein-ligand binding sites, enabling design aimed at exploiting all potential interactions. NMR spectroscopy, by measuring the propensity of the free ligand to adopt the required bioactive form, can inform and inspire ligand rigidification 
strategies, to minimize adverse energy contributions from nonbioactive states in solution. For example, LaPlante et al. conducted a detailed study of free and bound forms of NS3 protease inhibitors [11], and experimentally demonstrated that increases in binding affinity upon methylation correlated with rigidification of the free ligand. They showed that bioactive conformations, observed by x-ray crystallography, for the most potent leads were highly populated in solution. Furthermore, they also highlighted that five molecules in clinical development and two recently marketed drugs share the same bioactive rigid peptidic core. In an elegant fragment-based drug discovery example, Tamanini et al. [5] designed potent inhibitors of XIAP, and used NMR structural information on the free ligand to demonstrate a stable folded conformation, consistent with optimal binding to the protein. In this case, ligand folding was stabilized by an intramolecular dipole-dipole interaction, which if too strong led to intrinsic chemical instability.

Free ligands have unique NMR proton chemical shift signatures, and these can be used to assess the degree of flexibility or rigidity of a molecule in solution. If a molecule has a dominant conformer in which one or more protons are subject to significant perturbation, for example, from an aromatic ring current, this should be observable in a $1 D$ NMR spectrum. If we combine this information with target affinity, we can often rationalize SAR which is independent of any specific ligand-protein interaction. A recent example was described by Johannes et al. [4] in their design of macrocyclic inhibitors of MCL1. In this case they used knowledge of anomalous proton NMR chemical shifts caused by anisotropic shielding to understand that their most potent macrocycle, containing a methylated amide, had a conformational lock which biased it towards adopting the bioactive conformation in solution. In contrast, the less potent desmethylated analog did not display the same diagnostic chemical shift linked to the bound conformation and was instead conformationally biased towards a nonbioactive population in solution. Evidence that the methylated analog was pre-organised in the bioactive conformation was also apparent when measuring the kinetics of binding. In this regard, the enhanced potency of the methylated analog was associated with tenfold faster onrate kinetics compared with the nonbioactive analog.

A key learning from multiple design campaigns supported by free ligand NMR solution structure, is that potent ligands very often have a dominant conformer in solution which is very close to the bioactive conformation. Furthermore, the availability of the free ligand conformational preference helps to rationalize SAR by disentangling the contribution of protein-ligand interactions from ligand conformational effects, enabling more effective structure-based design. Structural information of both the bound and free ligand are needed to fully rationalize SAR and consequently to focus design and synthetic effort.

As a technique, NMR conformational analysis of ligands is well established. However, for such analyses to impact the medicinal chemistry design strategy within a project, there needs to be the ready availability of an experimental free ligand conformational ensemble. In the past such data have typically not been routinely available during lead optimization largely owing to limitations associated with the integration of NMR data and the modeling of ligand conformations and NMR parameters which are all time-consuming activities. This has now changed with recent advances in experimental [15] and computational [16-18] platforms, which have made such detailed conformational analysis much more accessible. Moreover, with the advent of high performance computing, a combination of NMR data and theoretical models can be used to rapidly quantify the populations of all accessible conformers of a ligand in a relatively short timeframe.

In our experience, it is critical that free ligand conformational information is available to chemists within a timeframe consistent with 'design, make, test, analyze' cycle times of typical drug discovery projects. This enables such information to drive enhanced design hypotheses and ultimately impact chemistry productivity through a 'real-time' understanding of bound and free ligand conformational preferences for compounds that are being actively pursued within a project. In AstraZeneca, efforts are ongoing to automate and routinely integrate potency and simple proton NMR data with structural information to enhance the structure-based drug design paradigm, with the ultimate intent of accelerating project cycle times. The simple 1D NMR signature of the free ligand can synergize with measured potency data to rationalize positive and negative SAR trends and a medicinal chemist can then recognize these from routinely available data. The degree of ligand flexibility and how this translates into conformational populations in solution can be readily assessed through analysis of the 1D NMR spectrum. This in turn can enable an understanding of what entropic penalties are being made in transitioning from the solution state to the bound state. Similarly, if the ligand is biased towards a nonbioactive conformation, its NMR signature will no longer tally with that predicted for the bioactive conformation and this could help explain an unexpected loss of potency observed for a new design. Alternatively, if the free solution ensemble is fully in the bioactive state and the ligand exploits all possible interactions in the binding site, it is unlikely that further significant gains in potency 
can be made through incremental changes to the molecule. In this case, the design focus should shift either towards addressing other developability end points (i.e., optimizing pharmacokinetics) or if intrinsic potency remains an issue, making more significant changes to the molecule that may, for example, require a change to the underlying scaffold of the molecule.

As well as using this approach to optimize potency, there is also an opportunity to relate conformational preferences through to measurable physicochemical end points, such as exposed molecular polarity which ultimately has the potential to impact important properties (i.e., oral bioavailability. This possibility has been highlighted in a number of studies [19], and thus there is the potential to exploit experimental free ligand conformational data to aid the medicinal chemists in the optimization of physical properties consistent with drug-like molecules. In this respect, the utilization of free ligand conformational data on emerging modalities, such as proteolysis targeting chimeras (PROTACs) [20], which can be characterized as large small-molecules with a relatively high number of rotatable bonds, may help medicinal chemists rationalize and optimize potency as well as improve broader developability properties.

Finally, to derive synergy through the combination of x-ray structure and free ligand NMR conformations, and achieve a turnaround compatible with 'design, make, test, analyze' cycle times, it is crucial to establish a collaborative culture among the different disciplines involved in drug optimization. In AstraZeneca we have developed a highly interdisciplinary approach, whereby medicinal and computational chemists, NMR spectroscopists and crystallographers collaborate and integrate structural data into the SAR of synthesized compounds, to rationalize existing data and to predict the impact of conformational effects in design sets yet to be made. This enhanced approach to drug design, incorporating free ligand conformational analysis, is inspiring more refined design hypotheses and positively impacting project cycle times.

\section{Financial \& competing interests disclosure}

The authors have no relevant affiliations or financial involvement with any organization or entity with a financial interest in or financial conflict with the subject matter or materials discussed in the manuscript. This includes employment, consultancies, honoraria, stock ownership or options, expert testimony, grants or patents received or pending, or royalties.

No writing assistance was utilized in the production of this manuscript.

\section{References}

1 Boehr DD, Nussinov R, Wright PE. The role of dynamic conformational ensembles in biomolecular recognition. Nat. Chem. Biol. 5(11), 789-796 (2009).

2 Zheng Y, Tice CM, Singh SB. Conformational control in structure-based drug design. Bioorg. Med. Chem. Lett. 27(13), 2825-2837 (2017).

3 Lawson ADG, MacCoss M, Heer JP. Importance of rigidity in designing small molecule drugs to tackle protein-protein interactions (PPIs) through stabilization of desired conformers. J. Med. Chem. 61(10), 4283-4289 (2018).

4 Johannes JW, Bates S, Beigie C et al. Structure based design of non-natural peptidic macrocyclic Mcl-1 inhibitors. ACS Med. Chem. Lett. 8(2), 239-244 (2017).

5 Tamanini E, Buck IM, Chessari G et al. Discovery of a potent nonpeptidomimetic, small-molecule antagonist of cellular inhibitor of apoptosis protein 1 (cIAP1) and x-linked inhibitor of apoptosis protein (XIAP). J. Med. Chem. 60(11), 4611-4625 (2017).

6 Thepchatri P, Eliseo T, Cicero DO et al. Relationship among ligand conformations in solution, in the solid state, and at the Hsp90 binding site: geldanamycin and radicicol. J. Am. Chem. Soc. 129(11), 3127-3134 (2007).

7 Grimmer C, Moore TW, Padwa A et al. Antiviral atropisomers: conformational energy surfaces by NMR for host-directed myxovirus blockers. J. Chem. Inf. Model. 54(8), 2214-2223 (2014).

8 Corzana F, Cuesta I, Bastida A et al. Molecular recognition of aminoglycoside antibiotics by bacterial defence proteins: NMR study of the structural and conformational features of streptomycin inactivation by Bacillus subtilis aminoglycoside-6-adenyl transferase. Chem. Eur. J. 11(17), 5102-5113 (2005).

9 Di Fabio R, Pellacani A, Faedo S et al. Discovery process and pharmacological characterization of a novel dual orexin 1 and orexin 2 receptor antagonist useful for treatment of sleep disorders. Bioorg. Med. Chem. Lett. 21(18), 5562-5567 (2011).

10 Howard S, Amin N, Benowitz AB et al. Fragment-based discovery of 6 azaindazoles as inhibitors of bacterial DNA ligase. ACS Med. Chem. Lett. 4(12), 1208-1212 (2013).

11 LaPlante SR, Nar H, Lemke CT et al. Ligand bioactive conformation plays a critical role in the design of drugs that target the hepatitis C virus NS3 protease. J. Med. Chem. 57(5), 1777-1789 (2014).

12 Martins FA, Zeoly LA, Cormanich RA et al. Solution conformations for the flexible 1-chloro-1,1-difluoro-2-pentanol unveiled using multinuclear magnetic resonance. Tetrahedron 74(8), 880-883 (2018). 
13 Scott JS, Degorce SL, Anjum R et al. Discovery and optimization of pyrrolopyrimidine inhibitors of interleukin-1 receptor associated kinase 4 (IRAK4) for the treatment of mutant MYD88L265P diffuse large B-cell lymphoma. J. Med. Chem. 60(24), 10071-10091 (2017).

14 Georgsson J, Bergstroem F, Nordqvist A et al. GPR103 antagonists demonstrating anorexigenic activity in vivo: design and development of Pyrrolo[2,3-c]pyridines that mimic the C-terminal Arg-Phe Motif of QRFP26. J. Med. Chem. 57(14), 5935-5948 (2014).

15 Butts CP, Jones CR, Song Z et al. Accurate NOE-distance determination enables the stereochemical assignment of a flexible molecule arugosin C. Chem. Commun. 48(72), 9023-9025 (2012).

16 Troche-Pesqueira E, Anklin C, Gil RR et al. Computer-assisted 3D structure elucidation of natural products using residual dipolar couplings. Angew. Chem. Int. Ed. 56(13), 3660-3664 (2017).

17 Blundell CD, Packer MJ, Almond A. Quantification of free ligand conformational preferences by NMR and their relationship to the bioactive conformation. Bioorg. Med. Chem. 21(17), 4976-4987 (2013).

18 Blundell CD, Nowak T, Watson MJ. Measurement, interpretation and use of free ligand solution conformations in drug discovery. Prog. Med. Chem. 55, 45-147 (2016).

19 Over B, Matsson P, Tyrchan C et al. Structural and conformational determinants of macrocycle cell permeability. Nat. Chem. Biol. 12(12), 1065-1074 (2016).

20 Churcher I. Protac-induced protein degradation in drug discovery: breaking the rules or just making new ones? J. Med. Chem. 61(2), 444-452 (2018). 